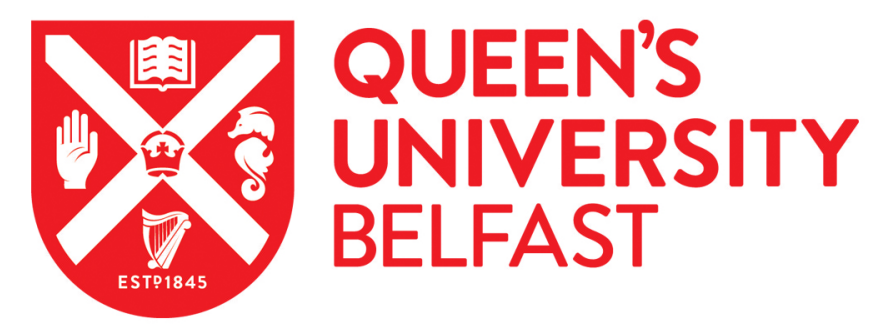

\title{
Virtual room acoustics using finite difference methods. How to model and analyse frequency-dependent boundaries?
}

Kowalczyk, K., \& Van Walstijn, M. (2008). Virtual room acoustics using finite difference methods. How to model and analyse frequency-dependent boundaries?. 1504-1509. Paper presented at 3rd IEEE International Symposium on Control, Communications and Signal Processing (ISCCSP 2008), St Julians, Malta.

\section{Document Version:}

Peer reviewed version

Queen's University Belfast - Research Portal:

Link to publication record in Queen's University Belfast Research Portal

\section{General rights}

Copyright for the publications made accessible via the Queen's University Belfast Research Portal is retained by the author(s) and / or other copyright owners and it is a condition of accessing these publications that users recognise and abide by the legal requirements associated with these rights.

Take down policy

The Research Portal is Queen's institutional repository that provides access to Queen's research output. Every effort has been made to ensure that content in the Research Portal does not infringe any person's rights, or applicable UK laws. If you discover content in the Research Portal that you believe breaches copyright or violates any law, please contact openaccess@qub.ac.uk. 


\title{
Virtual Room Acoustics using Finite Difference Methods. How to Model and Analyse Frequency-Dependent Boundaries?
}

\author{
Konrad Kowalczyk and Maarten van Walstijn \\ Sonic Arts Research Centre (SARC) \\ School of Electronics, Electrical Engineering and Computer Science \\ Queen's University, Belfast, Northern Ireland \\ \{kkowalczyk01, m.vanwalstijn\}@qub.ac.uk
}

\begin{abstract}
In this paper, we present new methods for constructing and analysing frequency-dependent boundaries in room acoustic modelling with the use of finite difference time domain (FDTD) techniques. Novel FDTD formulations of simple locally reacting wall models with complex impedance are proposed and analysed in terms of pressure wave reflectance for different wall impedances and angles of incidence. The analysis is done using both numerical experiments and analytic evaluation.

For the numerical experiments, a compact implicit scheme of 4th-order accuracy is used for updating the room interior grid points, the results of which are analysed in both time and frequency domains. The simulation results show that the 2D frequency-dependent locally reacting wall models adhere well to their theoretical counterparts, particularly at low frequencies. Furthermore, they validate the analytic evaluation method, which paves the way for using either method as a tool for analysis of numerical reflectance.
\end{abstract}

Index Terms-Acoustic reflection, acoustic impedance, acoustic signal processing, FDTD methods, waveguides

\section{INTRODUCTION}

Finite Difference (FD) is a modelling technique that can be applied to room acoustic simulations [1]. Recent research efforts have been focused on developing accurate FD approximations of realistic boundaries. Most of the boundary models available in the literature are based on a 1D approach [2], [3]. However, this leads to significant errors in the reflectance phase and amplitude [4]. For a physically more correct impedance boundary formulation, the boundary should be included in the medium, which can be obtained by combining the $1 \mathrm{D}$ boundary condition with the multi-dimensional (i.e. 2D or 3D) wave equation. Since frequency-dependent absorption occurs at realistic boundaries, the amplitude and phase of the reflected sound wave differ from those of the incident wave [5]. Instead of combining the FD implementation for the room interior with filters at boundaries [6], direct incorporation of the wall impedance in the boundary condition can be used. Alternative models rely on modelling the wave propagation in the wall [7]. However, previous studies [1] have suggested that there is no significant difference between the locally and

This research has been supported by the European Social Fund. non-locally reacting wall models used in FD room acoustic simulations.

Sec. II presents the numerical formulation of the 2D FD frequency-dependent locally reacting wall models. In sec. III, an analytic evaluation method for the analysis of the numerical reflectance in 2D/3D acoustic spaces is presented. The results of numerical experiments and the numerical boundary analysis are discussed in sec. IV and V, respectively. Considerations here are restricted to 2D modelling; analogous 3D models will be dealt with in a subsequent paper.

\section{FREQUENCY-DEPENDENT BOUNDARIES IN FD METHOD}

Sound wave reflection from a wall can be modelled as a locally reacting wall (LRW), where the normal component of the particle velocity at the surface of the wall depends on the sound pressure in front of the wall element and not on pressure in front of neighbouring elements [5]. As such, it provides a compact model for simulating specular wall reflections. In terms of pressure only this may be stated as [4]

$$
\frac{\partial p}{\partial t}=-c \frac{Z}{\rho c} \frac{\partial p}{\partial x}
$$

where $p$ denotes acoustic pressure, $c$ is the sound velocity and $Z / \rho c$ is the normalised wall impedance, also known as the specific acoustic impedance. The multi-dimensional FD formulation of a locally reacting wall is obtained by combining the discretised boundary condition (1) with the discrete multidimensional wave equation [4]. The reflectance $R$ is related to the specific acoustic impedance by [5]

$$
R=\frac{\frac{Z}{\rho c} \cos \theta-1}{\frac{Z}{\rho c} \cos \theta+1},
$$

where $\theta$ denotes the angle of incidence.

Real acoustic boundaries are generally frequencydependent, even in narrow frequency bands. Among the simplest of such boundaries, we can distinguish two types. The first one represents a thin absorbing layer stretched on the much harder boundary; seat, floor and wall coverings are good examples. The second type represents heavy porous 
layers such as curtains or light non stiff walls. More complex boundaries can be formulated as a superposition of these two types [1].

\section{A. Mass boundary}

The first boundary type, representing a nonporous layer hung in front of the rigid wall [5], defines an impedance as $Z=R+M s$, where $R$ denotes resistance, $M$ is the mass per unit area, and $s$ is the Laplace variable. Inserting such an impedance into Eq. (1) yields the mass boundary condition

$$
\frac{\partial p}{\partial t}=-c\left(\frac{R}{\rho c} \frac{\partial p}{\partial x}+\frac{M}{\rho c} \frac{\partial p^{2}}{\partial t \partial x}\right)
$$

The FD discrete boundary is obtained by approximating the first-order derivatives in time and space domains with centered FD operators. In order to keep the boundary formula explicit the mixed second-order derivative in Eq. (3) can be approximated with the backward Euler method

$$
\begin{aligned}
\frac{\partial^{2} p}{\partial t \partial x} & =\frac{\left.\frac{\partial p}{\partial x}\right|^{n}-\left.\frac{\partial p}{\partial x}\right|^{n-1}}{T} \\
& =\frac{1}{2 T X}\left(p_{l+1, m}^{n}-p_{l-1, m}^{n}-p_{l+1, m}^{n-1}+p_{l-1, m}^{n-1}\right),
\end{aligned}
$$

where $X$ denotes grid spacing, $T$ is a time step, $p_{l}^{n}$ is the pressure update variable, $l$ and $m$ denote spatial indexes and $n$ is a time index, respectively. Such an asymmetrical approximation in the time domain is the weakest point of the FD discretisation of a mass boundary condition and will lead to small phase errors in the reflectance. Nevertheless, the numerical error is less severe than for an asymmetrical approximation in the space domain, which has been proposed in [1]. If we write out the discrete version of Eq. (3) for the point lying outside of the modelled space, also referred to as a 'ghost point', the following formula results

$$
\begin{aligned}
p_{l+1, m}^{n} & =p_{l-1, m}^{n}+\frac{1}{\lambda\left(a_{1}+a_{2}\right)}\left(p_{l, m}^{n-1}-p_{l, m}^{n+1}\right) \\
& +\frac{a_{2}}{a_{1}+a_{2}}\left(p_{l+1, m}^{n-1}-p_{l-1, m}^{n-1}\right),
\end{aligned}
$$

where $\lambda=c T / X$ is the Courant number and the parameters $a_{1}$ and $a_{2}$ are given in Table I. Next, the update formula for the boundary node is obtained by substituting for the ghost point in a discrete $2 \mathrm{D}$ wave equation

$$
\begin{aligned}
p_{l, m}^{n+1} & =\lambda^{2}\left(p_{l+1, m}^{n}+p_{l-1, m}^{n}+p_{l, m+1}^{n}+p_{l, m-1}^{n}\right) \\
& +2\left(1-2 \lambda^{2}\right) p_{l, m}^{n}-p_{l, m}^{n-1},
\end{aligned}
$$

with the boundary condition given by Eq. (5), which yields

$$
\begin{aligned}
p_{l, m}^{n+1} & =\left[\lambda^{2}\left(2 p_{l-1, m}^{n}+p_{l, m+1}^{n}+p_{l, m-1}^{n}\right)\right. \\
& +2\left(1-2 \lambda^{2}\right) p_{l, m}^{n}+\left(\frac{\lambda}{a_{1}+a_{2}}-1\right) p_{l, m}^{n-1} \\
& \left.+\frac{\lambda^{2} a_{2}}{a_{1}+a_{2}}\left(p_{l+1, m}^{n-1}-p_{l-1, m}^{n-1}\right)\right] /\left(1+\frac{\lambda}{a_{1}+a_{2}}\right) .
\end{aligned}
$$

This boundary formulation requires updating the boundary node and the ghost point at each time step according to Eq. (7) and Eq. (5), respectively. Only one past value needs to be stored at the ghost point.
TABLE I

COMPLEX IMPEDANCE PARAMETERS.

\begin{tabular}{|c|c|}
\hline Parameter & Description \\
\hline \hline$a_{1}=\frac{R}{\rho c}$ & Specific resistance \\
\hline$a_{2}=\frac{M}{T \rho c}$ & Specific mass divided by $\mathrm{T}$ \\
\hline$a_{3}=\frac{T K}{2 \rho c}$ & Specific spring constant multiplied by half of $\mathrm{T}$ \\
\hline$a_{4}=a_{1}+a_{2}+a_{3}$ & Sum of the previous parameters \\
\hline
\end{tabular}

\section{B. Spring boundary}

The second absorbing boundary type, representing materials such as a thin porous layer of fabric hung on a rigid wall [5], has the following impedance $Z=R+K / s$, where $K$ denotes the spring constant. The continuous spring boundary condition is given by

$$
\frac{\partial p}{\partial t}=-c\left(\frac{R}{\rho c} \frac{\partial p}{\partial x}+\frac{K}{\rho c} \int_{-\infty}^{t} \frac{\partial p}{\partial x} d t\right) .
$$

All first-order derivatives in Eq. (8) are approximated with centered FD operators. As for a numerical integration method, we propose the use of a composite trapezoidal rule with subintervals equal to time steps because of the method's accuracy and good phase properties. Conversely, the use of the backward Euler integration, which was applied in [1], would result in a less correct reflectance phase and amplitude. Trapezoidal integration is mathematically equivalent to the bilinear transform $s=\frac{2}{T} \frac{1-z^{-1}}{1+z^{-1}}$ applied to $y=x / s$, which yields

$$
y^{n}=T \frac{x^{n}+x^{n-1}}{2}+y^{n-1},
$$

Consequently, numerical integration is given by

$$
\frac{K}{\rho c} \int_{-\infty}^{t} p_{l, m}^{n} d t=\frac{T K}{2 \rho c} \sum_{i=-\infty}^{n}\left(p_{l, m}^{i}+p_{l, m}^{i-1}\right) .
$$

Finally, the discrete version of the spring boundary condition for a ghost point becomes

$$
\begin{aligned}
p_{l+1, m}^{n} & =p_{l-1, m}^{n}+\frac{1}{\lambda\left(a_{1}+a_{3}\right)}\left(p_{l, m}^{n-1}-p_{l, m}^{n+1}\right) \\
& +\frac{a_{3}}{a_{1}+a_{3}}\left(p_{l-1, m}^{n-1}-p_{l+1, m}^{n-1}\right)+\frac{a_{3}}{a_{1}+a_{3}} S^{n-1}
\end{aligned}
$$

where the parameters $a_{1}$ and $a_{3}$ are given in Table I. The new variable $S^{n-1}$ is introduced for storage of the result of summation 'up to now' according to the formula

$$
S^{n-1}=p_{l-1, m}^{n-1}+p_{l-1, m}^{n-2}-p_{l+1, m}^{n-1}-p_{l+1, m}^{n-2}+S^{n-2} .
$$

Substituting for the ghost point in the respective discrete wave equation (6) with the boundary condition (11), the update formula for the spring boundary node yields

$$
\begin{aligned}
p_{l, m}^{n+1} & =\left[\lambda^{2}\left(2 p_{l-1, m}^{n}+p_{l, m+1}^{n}+p_{l, m-1}^{n}\right)+2\left(1-2 \lambda^{2}\right) p_{l, m}^{n}\right. \\
& +\left(\frac{\lambda}{a_{1}+a_{3}}-1\right) p_{l, m}^{n-1}+\frac{\lambda^{2} a_{3}}{a_{1}+a_{3}}\left(p_{l-1, m}^{n-1}-p_{l+1, m}^{n-1}\right) \\
& \left.+\frac{\lambda^{2} a_{3}}{a_{1}+a_{3}} S^{n-1}\right] /\left(1+\frac{\lambda}{a_{1}+a_{3}}\right) .
\end{aligned}
$$


This boundary formulation requires the update of the boundary node, the ghost point and the sum up to now at each time step according to Eq. (13), Eq. (11) and Eq. (12), respectively. However, only one previous value needs to be stored for both the ghost point and the sum value.

\section{Combined impedance boundary}

Some realistic acoustic boundaries might require a more complex impedance; parquet is a good example. Another example is a porous layer of fabric stretched in front of the rigid wall, to which the second layer of nonporous material hung immediately in front of the first layer is added [5]. The resulting boundary condition for a combined impedance $Z=R+M s+K / s$ amounts to

$$
\frac{\partial p}{\partial t}=-c\left(\frac{R}{\rho c} \frac{\partial p}{\partial x}+\frac{M}{\rho c} \frac{\partial p^{2}}{\partial x \partial y}+\frac{K}{\rho c} \int_{-\infty}^{t} \frac{\partial p}{\partial x} d t\right),
$$

The derivation relies on the combination of the previously presented boundary models. Hence, the node at the boundary is updated according to

$$
\begin{aligned}
p_{l, m}^{n+1} & =\left[\lambda^{2}\left(2 p_{l-1, m}^{n}+p_{l, m+1}^{n}+p_{l, m-1}^{n}\right)+2\left(1-2 \lambda^{2}\right) p_{l, m}^{n}\right. \\
& +\left(\frac{\lambda}{a_{4}}-1\right) p_{l, m}^{n-1}+\frac{\lambda^{2}\left(a_{3}-a_{2}\right)}{a_{4}}\left(p_{l-1, m}^{n-1}-p_{l+1, m}^{n-1}\right) \\
& \left.+\frac{\lambda^{2} a_{3}}{a_{4}} S^{n-1}\right] /\left(1+\frac{\lambda}{a_{4}}\right),
\end{aligned}
$$

and the update formula for the ghost point is given by

$$
\begin{aligned}
p_{l+1, m}^{n} & =p_{l-1, m}^{n}+\frac{1}{\lambda a_{4}}\left(p_{l, m}^{n-1}-p_{l, m}^{n+1}\right)+ \\
& +\frac{a_{3}-a_{2}}{a_{4}}\left(p_{l-1, m}^{n-1}-p_{l+1, m}^{n-1}\right)+\frac{a_{3}}{a_{4}} S^{n-1},
\end{aligned}
$$

where the parameters $a_{1}, a_{2}, a_{3}$ and $a_{4}$ are given in Table I, and the sum $S$ is given by Eq. (12). Similarly to the spring boundary, Eq. (15), Eq. (16) and Eq. (12) need to be computed at each time step.

\section{ANALYTiC EVALUATION METHOD}

In this section, a novel method for analytic evaluation of the numerical reflection of multi-dimensional boundaries is presented. Due to the limited space, this paper outlines briefly the concept behind the numerical boundary analysis (NBA) and presents the final formula for the numerical reflectance of the combined impedance model.

Consider a wall normal to the rectangular coordinate system in the $x-y$ plane, where the wall is located at $x=0$. An incident wave propagating at any oblique angle of incidence $\theta$ in a positive $x$-direction in such a system can be expressed as [5]

$$
p=P_{0} e^{j \omega t} e^{-j k(x \cos \theta+y \sin \theta)},
$$

where $P_{0}$ is the incident wave amplitude and $k$ denotes the wave number. As for the reflected wave, the sign is reversed and the pressure amplitude is multiplied by the reflection factor. Hence the total sound pressure in the standing wave in the plane of the locally reacting wall can be obtained by adding both incident and reflected sound pressure values and setting $x=0$, which in the discrete space-time domain takes the following form

$$
p_{l, m}^{n}=P_{0} e^{j \omega n T} e^{-j k m X \sin \theta}\left(e^{-j k l X \cos \theta}+\widehat{R} e^{j k l X \cos \theta}\right) .
$$

Eq. (18) can be used as a basis for deriving other discrete pressure values in the respective boundary equation. Some example expressions are given below

$$
\begin{aligned}
p_{l, m}^{n+1} & =e^{j \omega T} p_{l, m}^{n}=e^{s T} p_{l, m}^{n}=z p_{l, m}^{n}, \\
p_{l-1, m}^{n}= & P_{0} e^{j \omega n T} e^{-j k m X \sin \theta} \\
& \cdot\left(e^{-j k(l-1) X \cos \theta}+\widehat{R} e^{j k(l-1) X \cos \theta}\right) .
\end{aligned}
$$

Next, we substitute such discrete pressure values in the discrete boundary equation and set $l=0$, which corresponds to $x=0$ at a boundary. Finally, the analytic evaluation method relies on solving for the numerical reflectance $\widehat{R}$. In case of the combined impedance boundary condition given by Eq. (15) the following formula results

$$
\begin{aligned}
\widehat{R}(z, \theta) & =-\left\{\left(1+\frac{\lambda}{a_{4}}\right) z-\left[2 \lambda^{2} A+\lambda^{2}\left(B+B^{-1}\right)\right.\right. \\
& \left.+\quad 2\left(1-2 \lambda^{2}\right)\right]+\left[1-\frac{\lambda}{a_{4}}+\frac{\lambda^{2} a_{2}}{a_{4}}\left(A-A^{-1}\right)\right. \\
& \left.\left.+\frac{\lambda^{2} a_{3}}{a_{4}}\left(A^{-1}-A\right) \frac{2}{1-z^{-1}}\right] z^{-1}\right\} \\
& /\left\{\left(1+\frac{\lambda}{a_{4}}\right) z-\left[2 \lambda^{2} A^{-1}+\lambda^{2}\left(B+B^{-1}\right)\right.\right. \\
& \left.+2\left(1-2 \lambda^{2}\right)\right]+\left[1-\frac{\lambda}{a_{4}}+\frac{\lambda^{2} a_{2}}{a_{4}}\left(A^{-1}-A\right)\right. \\
& \left.\left.+\frac{\lambda^{2} a_{3}}{a_{4}}\left(A-A^{-1}\right) \frac{2}{1-z^{-1}}\right] z^{-1}\right\},
\end{aligned}
$$

where $A=e^{j k X \cos \theta}$ and $B=e^{j k X \sin \theta}$. Eq. (21) can be used to predict accurately the numerical reflectance for any value of the impedance and angle of incidence. This formula is valid for up to a quarter of the sample rate because the discretised 2D wave equation has a cut-off at $0.25 f_{s}$ in axial directions (i.e. there are no resonances for the wave propagation in axial directions for the 2D rectilinear scheme). The amplitude of the analytically evaluated numerical reflectance (solid lines) in the frequency domain for the boundary models derived in this paper is presented in figures 3, 4, and 5, respectively.

Note that the evaluation method requires the same multidimensional FD scheme for the boundary and the medium inside of the modelled space. Consequently, this method is not suitable for the evaluation of the $1 \mathrm{D}$ boundaries in a $2 \mathrm{D} / 3 \mathrm{D}$ context. The great advantage of the analytic evaluation method is that it produces results which are deprived of the numerical artefacts associated with the numerical experiments due to dispersion and wave truncation errors. Consequently, the NBA method can be successfully used to evaluate the numerical reflectance instead of undertaking numerous numerical experiments. 


\section{NUMERICAL EXPERIMENTS}

\section{A. Test setup}

A number of simulations were carried out to analyse the performance of the three types of frequency-dependent boundary conditions of a locally reacting wall derived in section II. A fourth-order accurate compact implicit scheme [8] with 1800x1400 junctions was used for the implementation of the interior of the room in order to obtain the wavefront that was as flat as possible. The size of the room and the simulation time (2000 samples at the sample rate of $4 \mathrm{kHz}$ ) were set in such a way that only the reflections from the investigated boundary could reach a receiver position. Furthermore, the simulation time had to be sufficiently long so that the whole wavelet could reach a receiver position. Each test consisted of two simulations, in which a sharp impulse was injected into a mesh point. In each test, the source position was chosen so that (1) a constant distance of 400 grid points from the centre of the investigated wall was preserved, and (2) the incident waves at the following angles of incidence $\theta=0^{\circ}, 15^{\circ}, 30^{\circ}, 45^{\circ}, 60^{\circ}, 75^{\circ}$, resulted. In the first simulation, the reflected signal $x_{f}$ was measured at a receiver position located at the same distance from the centre of the wall as a source; $x_{f}$ inevitably included the 'direct sound'. The wall was removed in the second simulation and two signals were measured: the direct sound $x_{d}$ at the same receiver position and the freefield signal $x_{i}$ at the mirror location of the receiver. The isolated reflected signal $x_{r}$ was obtained by $x_{r}=x_{f}-x_{d}$.

For the time domain analysis, the ideal time domain signal was obtained as a time domain convolution of the freefield signal $x_{i}$ and the signal obtained from the inverse Laplace transform of the theoretical reflectance given by Eq. (2). The choice of the Laplace transform was made to avoid the characteristic ripples due to the inverse Fourier transforms. In order to reduce truncation, the resulting signal was convolved with a 41-tap low pass FIR filter with a normalised cut-off frequency of 0.25 .

For the frequency domain plots, the numerical reflectance was defined as the deconvolution of $x_{r}$ and $x_{i}$ and the theoretical reflectance given by Eq. (2) was used as a reference. Furthermore, all the measured signals were windowed with the use of the right half of the Hanning window to reduce signal truncation errors.

The values of parameters related to specific resistance, mass and spring were chosen to illustrate the numerical performance of the boundary models presented in this paper; they were not intended to represent realistic boundaries.

\section{B. Time domain analysis}

A reflected sound wave has both phase and amplitude which differ from those of an incident wave if the boundary has a complex impedance. In this section, the phase of numerical boundary models is analysed through the time domain comparison of the reflected signals obtained from numerical experiments with the ideal reflection signals.

Two representative results are illustrated in Fig. 1 and Fig. 2 , namely the spring boundary with $a_{1}=9$ and $a_{3}=1$

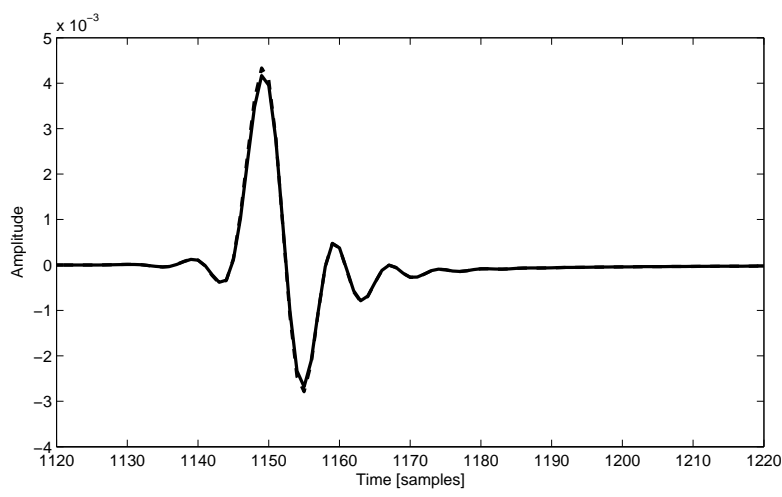

Fig. 1. Reflected signal (solid line) plotted against the theoretical reflection signal (dashed line) for the $2 \mathrm{D}$ spring boundary model at the angle of incidence $\theta=60^{\circ}$, where $a_{1}=9$ and $a_{3}=1$.

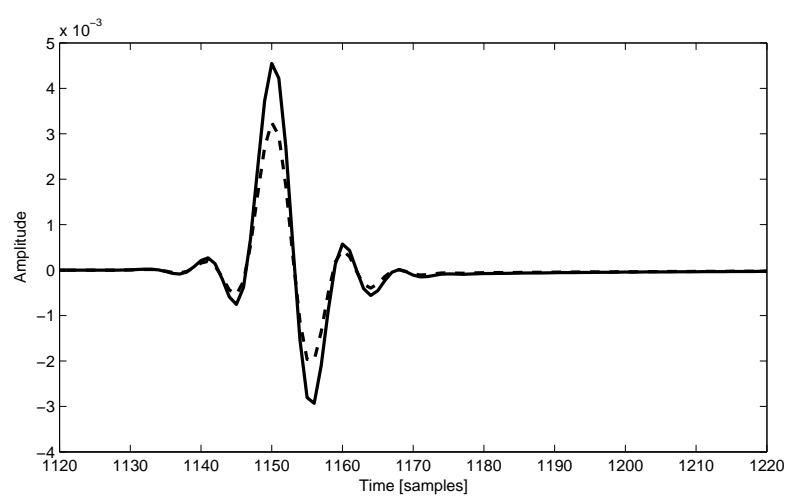

Fig. 2. Reflected signal (solid line) plotted against the theoretical reflection signal (dashed line) for the $2 \mathrm{D}$ combined impedance boundary model at the angle of incidence $\theta=45^{\circ}$, where $a_{1}=9, a_{2}=8$, and $a_{3}=0.5$.

for an incident angle $\theta=60^{\circ}$ and the combined impedance boundary with $a_{1}=9, a_{2}=8, a_{3}=0.5$ for incident angle $\theta=45^{\circ}$; respectively. As depicted in Fig. 1, the spring boundary model preserves the phase perfectly and the amplitude is well matched. Such a correct phase characteristic is down to the trapezoidal integration method which has excellent phase properties. Consequently, even at very high angles of incidence the phase is correct, which is not the case for 1D boundary models [4]. Fig. 2 confirms that in the case of the combined impedance boundary the phase is also preserved for high angles of incidence, however the amplitude is rather overestimated. Slight misadjustments at high incident angles may occur due to the use of the backward Euler method in the time domain approximation of the mixed derivative.

\section{Reflectance magnitude analysis}

In this section, the frequency-dependent reflectance amplitude is analysed in the frequency domain. The reflectance magnitudes of the three types of boundary conditions derived in section II are illustrated in figures 3, 4, and 5, for a variety of parameters and incident angles. All figures plot the reflectance obtained from numerical experiments (dashed lines), the reflectance predicted with the use of the analytic 

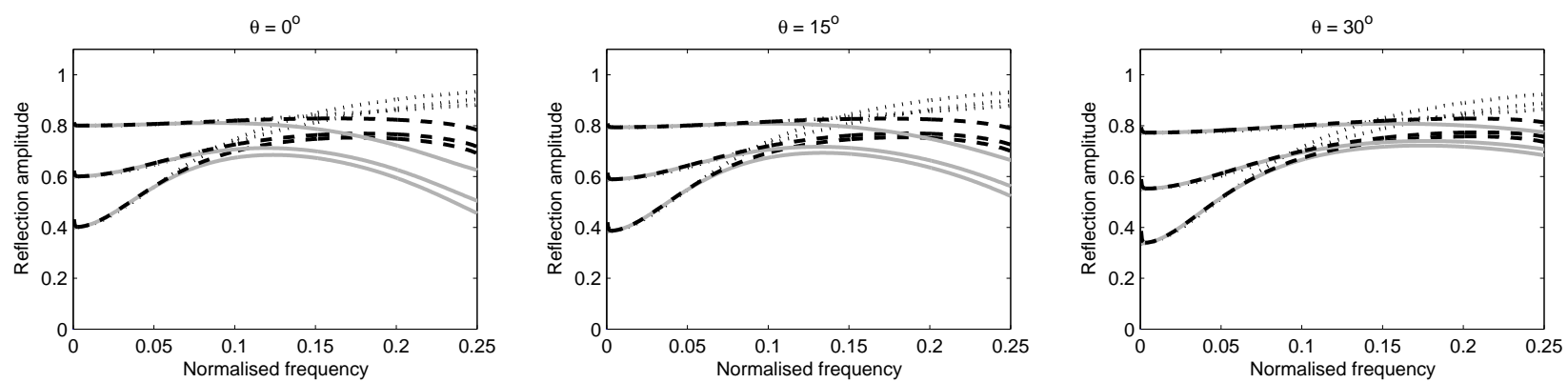

$\theta=45^{\circ}$
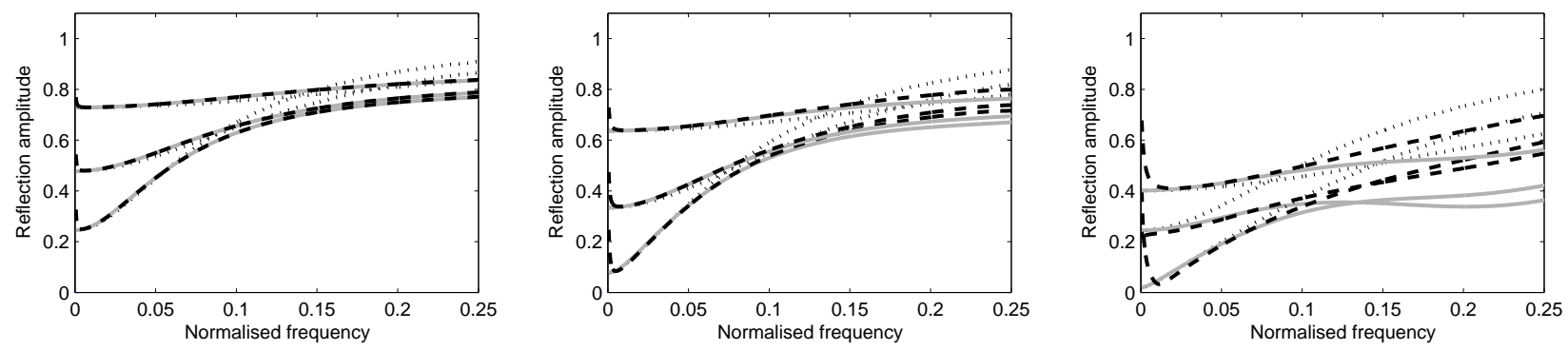

Fig. 3. Numerical reflection of the mass boundary for the following values of parameters $a_{1}=\frac{7}{3}, 4,9, a_{2}=5$ and angles of incidence $\theta=0^{\circ}, 15^{\circ}, 30^{\circ}, 45^{\circ}, 60^{\circ}, 75^{\circ}$. Dashed lines denote the reflectance obtained from numerical measurements, dotted lines denote the theoretical value of the reflection coefficient and grey solid lines represent numerical reflectance obtained from the analytic evaluation method.
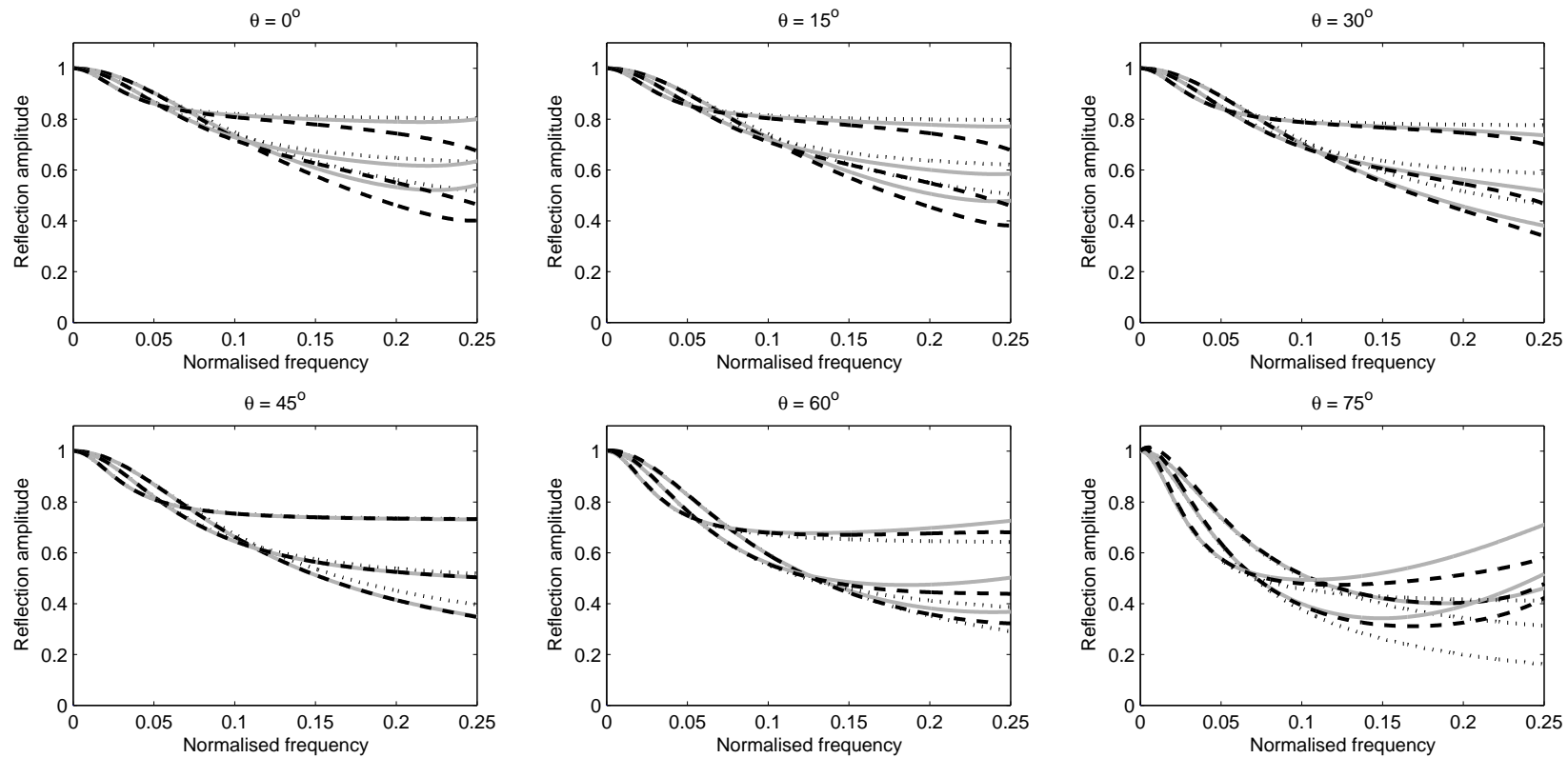

Fig. 4. Numerical reflection of the spring boundary for the following values of parameters $a_{1}=\frac{7}{3}, 4,9, a_{3}=1$ and angles of incidence $\theta=0^{\circ}, 15^{\circ}, 30^{\circ}, 45^{\circ}, 60^{\circ}, 75^{\circ}$. Dashed lines denote the reflectance obtained from numerical measurements, dotted lines denote the theoretical value of the reflection coefficient, and grey solid lines represent numerical reflectance obtained from the analytic evaluation method.

evaluation method (solid lines) and the theoretical reflection (dotted lines).

The theoretical reflection coefficient is matched well in general for up to a quarter of the sample rate for all the boundary models; in particular for a spring model as shown in Fig. 4. The numerical reflectance differs the most at normal incidences, which coincides with the fact that the numerical dispersion of the $2 \mathrm{D}$ rectilinear scheme is the strongest in axial direction. Furthermore, the numerical reflectance adheres well at low frequencies for all models, which is a desirable feature of FD models of rooms. The discrepancy near DC $(\omega=0)$, particularly visible at very high angles of incidence, is due to truncation error and the waves being not perfectly plane at the point of reflection. It should be stressed that the theoretical reflection coefficient was derived for plane waves, whereas in the experiments a spherical wave was used 


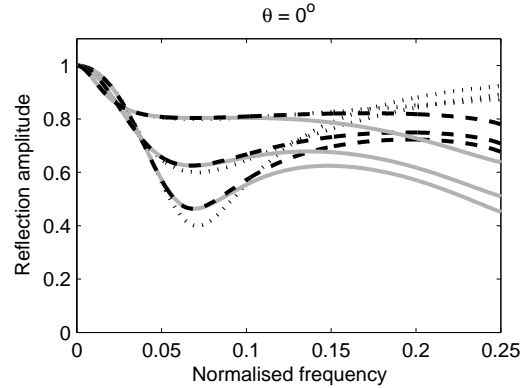

$\theta=45^{\circ}$

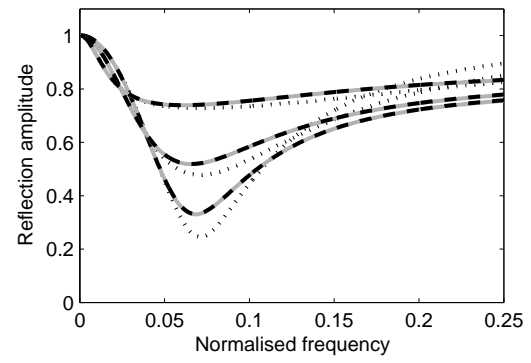

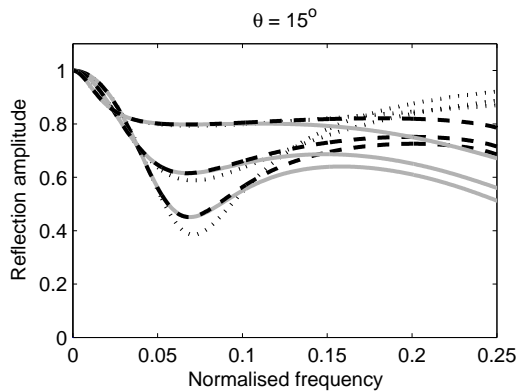

$\theta=60^{\circ}$

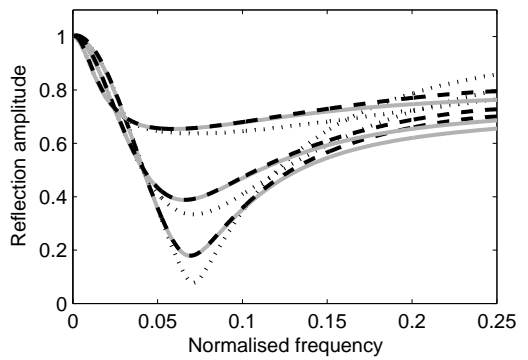

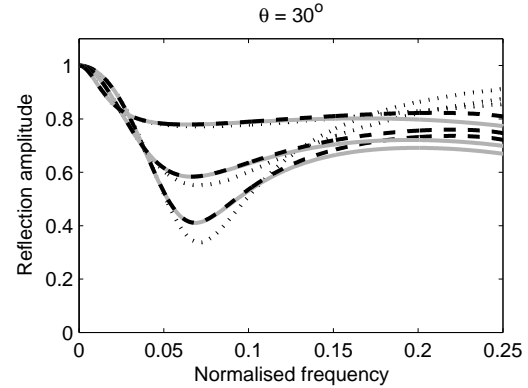

$\theta=75^{\circ}$

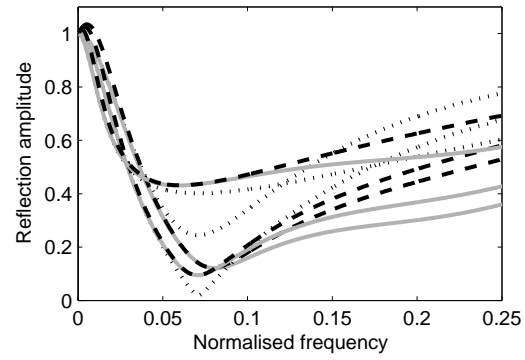

Fig. 5. Numerical reflection of a combined impedance boundary for the following values of parameters $a_{1}=\frac{7}{3}, 4,9, a_{2}=5, a_{3}=1$ and angles of incidence $\theta=0^{\circ}, 15^{\circ}, 30^{\circ}, 45^{\circ}, 60^{\circ}, 75^{\circ}$. Dashed lines denote the reflectance obtained from numerical measurements, dotted lines denote the theoretical value of the reflection coefficient, and grey solid lines represent numerical reflectance obtained from the analytic evaluation method.

for the excitation. Even though the curvature of a spherical wavefront after traveling a long distance before hitting the wall can be neglected, a minor discrepancy remains. The analytic evaluation plots confirm that a sudden outstrip near DC does not really occur for these boundary models.

\section{NUMERICAL BOUNDARY ANALYSIS RESULTS}

The correctness of the NBA is confirmed by the perfect match of the measured and evaluated reflectance in figures 3, 4 and 5 for the angle of incidence $\theta=45^{\circ}$ at which both the rectilinear scheme used in analytic evaluation formula derivation and the compact implicit scheme used for the room interior in numerical tests have no dispersion. There is also a good match for all possible angles of incidence and impedance values at low frequencies, where almost no numerical error occurs. In addition, the difference between the simulated and analytically evaluated numerical reflectance at high frequencies is due only to using a different scheme for the room interior. A closer match of the reflectance obtained from experiments than from NBA indicates that, despite the scheme discontinuity, a combination of the ADI method with these three boundary models leads to a more accurate numerical reflectance.

\section{CONCLUSION}

The new multi-dimensional frequency-dependent locally reacting wall models preserve the phase well even at high angles of incidence. Furthermore, the reflectance magnitude adheres well at low frequencies for all presented models, which is not the case for the $1 \mathrm{D}$ termination of multi-dimensional mesh simulations [4]. Consequently, such models provide a improved numerical formulation of frequency-dependent boundaries that can be applied in FD simulations of acoustic spaces.

In addition, an analytic evaluation method has been presented which can be used to predict the numerical reflectance for any impedance and angle of incidence in a fast and reliable way. As such, it provides a valuable tool for numerical reflectance analysis since the reflectance is deprived of artefacts which result from numerical experiments.

\section{REFERENCES}

[1] D. Botteldooren, "Finite-difference time-domain simulation of lowfrequency room acoustic problems," J. Acoustical Society America, vol. 98, no. 6, pp. 3302-3308, 1995.

[2] L. Savioja, T. Rinne, and T. Takala, "Simulation of room acoustics with a 3-D finite difference mesh," Proc. Int. Computer Music Conf. (ICMC), pp. 463-466, September 1994, Aarhus, Denmark.

[3] J. Huopaniemi, L. Savioja, and M. Karjalainen, "Modelling of reflections and air absorption in acoustical spaces - a digital filter design approach," Proc. IEEE Workshop on Appl. of Signal Processing to Audio and Acoustics (WASPAA), October 1997.

[4] K. Kowalczyk and M. van Walstijn, "Formulation of a locally reacting wall in Finite Difference modelling of acoustic spaces," Proc. Int. Symp. Room Acoustics (ISRA), pp. 1-6, September 2007, Seville, Spain.

[5] H. Kuttruff, Room acoustics, Applied Science Publishers Ltd, London, 1973.

[6] D.T. Murphy and M. Beeson, "The KW-boundary hybrid digital waveguide mesh for room acoustics applications," IEEE Transactions on Speech and Audio Processing, vol. 12, no. 2, pp. 552-564, 2007.

[7] A. Kelloniemi, "Improved adjustable boundary cindition for the 2D digital waveguide mesh," Proc. Int. Conf. Digital Audio Effects (DAFX'05), September 2005.

[8] K. Kowalczyk and M. van Walstijn, "On-line simulation of 2D resonators with reduced dispersion error using compact implicit finite difference methods," Proc. IEEE Int. Conf. Acoustics, Speech and Signal Processing (ICASSP), vol. 1, pp. 285-288, April 2007, Honolulu, Hawaii. 